\title{
Modeling RHIC Linear Chromaticity with Sextupole Components in the Arc Main Dipoles
}

\author{
Y. Luo, A. Jain, W. Fischer, S. Tepikian, D. Trbojevic
}

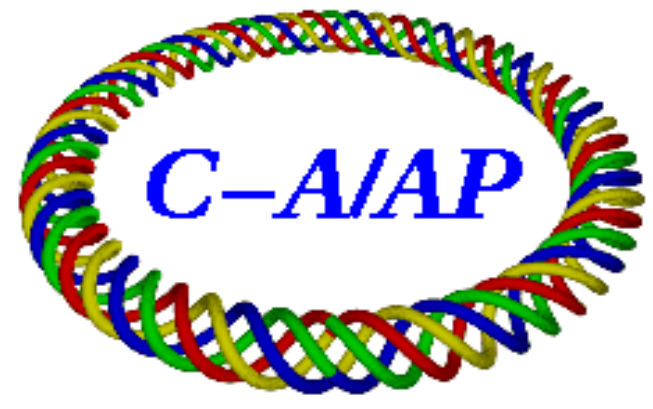

Collider-Accelerator Department Brookhaven National Laboratory Upton, NY 11973 
$\mathrm{CAD} / \mathrm{AP} / 276$

April 30, 2007

\title{
Modeling RHIC linear chromaticity with sextupole components in the arc main dipoles
}

\author{
Y. Luo, A. Jain, W. Fischer, S. Tepikian, and D. Trbojevic \\ Brookhaven National Laboratory, Upton, NY 11973, USA
}

The sextupole components in the arc main dipoles complicate predications of the linear chromaticities in the Relativistic Heavy Ion Collider (RHIC). In this article, the measured linear chromaticity splits are modeled with the sextupole components in the arc main dipoles. The values for the equivalent average sextupole strength $\left(K_{2} L\right)_{\text {dipole, mean }}$ in the main dipoles are derived with an offline optics model at Blue ring injection, Blue ring store, and Yellow ring injection. The findings are compared to the $b_{2}$ estimates from the bench measurements of the magnetic field. Reasons for the discrepancy between them are suggested.

\section{Introduction}

It is difficult to predict the linear chromaticities, $Q^{\prime} \mathrm{s}$, for the RHIC rings with the online optics models. The major contribtion to the discrepancy between the $Q^{\prime}$ measurements and their predictions is the sextupole components in the arc main dipoles. Furthermore, at the injection energy, the sextupole component in the arc main dipole varies because of the persistent current effect [1].

Out of 288 arc main dipoles in the Blue and Yellow rings, only about $20 \%$ were bench measured in the superconducting state before going into the RHIC's tunnel. In the online optics model, the strengths for the sextupole components in the arc main dipoles are partly extracted from this limited number of bench measurements, and partly from warm-cold correlations and interpolations.

Tab. 1 shows one example of measured and predicted $Q^{\prime}$ s taken at the Blue ring injection [2]. With the $2004 \mathrm{Au}$ run online optics model, the error in predicating linear chromaticity is about $\mp 14$ units, while with the updated $2007 \mathrm{Au}$ run optics model, the error in predication is about $\mp 7$ units. The two online models differ only in the strength settings of the sextupole components in arc main dipoles.

Table 1: Example of measurements and predictions of $Q^{\prime}$ s at the Blue ring injection.

\begin{tabular}{lccc}
\hline \hline & Measurement & $\begin{array}{c}\text { Online Model } \\
\text { Au04 Version }\end{array}$ & $\begin{array}{c}\text { Online Model } \\
\text { Au07 Version }\end{array}$ \\
\hline Horizontal tune, $Q_{x}$ & 28.224 & 28.253 & 28.243 \\
Vertical tune, $Q_{y}$ & 29.238 & 28.230 & 28.241 \\
Linear horizontal chromaticity, $Q_{x}^{\prime}$ & -8.1 & -26.5 & -15.0 \\
Linear vertical chromaticity, $Q_{y}^{\prime}$ & -7.1 & 7.0 & -0.08 \\
\hline \hline
\end{tabular}

\section{Beam-based measurement and modeling}

\subsection{Modeling procedure}

In the following, we assume that the differences in the measured $Q^{\prime}$ s and their predictions from the bare optics are solely due to the sextupole components in the arc main dipoles. In the bare optics, there is no sextupole component in the arc main dipoles. The contributions from the interaction regions are ignored, as well as the optical deviations.

In our following offline optics model, we artificially split the arc main dipoles into two halves and insert one sextupole multipole between. Considering there are 144 arc main dipoles in each RHIC ring, it is 
accurate to use the average integrated strength $\left(K_{2} L\right)_{\text {dipole,mean }}$ of these sextupole multipoles to model the $Q^{\prime}$ measurements. In the following modeling, all the sextupole multipoles in the arc main dipole centers have the same strength, $\left(K_{2} L\right)_{\text {dipole, mean }}$.

In each case, with the offline optics model, we first reproduce the measured horizontal and vertical tunes by adjusting the main quadrupole strengths. Then, with the real strengths of chromatic sextupoles, we alter $\left(K_{2} L\right)_{\text {dipole,mean }}$ in the offline model to minimize $\chi$,

$$
\chi=\left|Q_{x, \text { meas. }}^{\prime}-Q_{x, \text { model }, b 2}^{\prime}\right|+\left|Q_{y, \text { meas. }}^{\prime}-Q_{y, \text { model }, b 2}^{\prime}\right| .
$$

In total, there are 24 chromatic sextupole families in each RHIC ring. They can be sorted into 2 families, which only allows the linear chromaticity correction, or into 8 families for the nonlinear chromaticity correction. For simplicity, in the following study, we use 2 chromatic sextupole families, SF and SD, to set $Q_{x, y}^{\prime}$.

\subsection{At the Blue ring injection}

At the $23 \mathrm{GeV} \mathrm{Au}$ injection, the $\beta^{*}$ s at the crossing points are $10 \mathrm{~m}$. The natural linear chromaticities are about -45. Tab. 2 shows two cases of $Q_{x, y}^{\prime}$ measurements and modeling at the Blue ring injection. Comparing the $Q^{\prime}$ predictions from the bare optics which has no sextupole components $\left(b_{2} \mathrm{~s}\right)$ in arc main dipoles, we have

$$
\begin{aligned}
& Q_{x, y, \text { meas. }}^{\prime} \approx Q_{x, y, \text { model }, \text { no-b2 }}^{\prime} \mp 20.0, \text { for } \text { Case } 1, \\
& Q_{x, y, \text { meas. }}^{\prime} \approx Q_{x, y, \text { model }, \text { no-b2 }}^{\prime} \mp 17.6, \text { for } \text { Case } 2 .
\end{aligned}
$$

Then, adjusting $\left(K_{2} L\right)_{\text {dipole, mean }}$ to minimize $\chi$, for Case 1 , we have $\left(K_{2} L\right)_{\text {dipole,mean }} \approx-0.054 \mathrm{~m}^{-2}$, and for Case 2, this value is about $-0.047 \mathrm{~m}^{-2}$.

\subsection{At the Yellow ring injection}

Tab. 3 shows two cases of $Q_{x, y}^{\prime}$ measurements and modeling at the Yellow ring injection, wherein

$$
\begin{aligned}
& Q_{x, y, \text { meas. }}^{\prime} \approx Q_{x, y, \text { model }, \text { no-b2 }}^{\prime} \mp 20.1, \text { for Case } 3, \\
& Q_{x, y, \text { meas. }}^{\prime} \approx Q_{x, y, \text { model }, \text { no-b2 }}^{\prime} \mp 19.8, \text { for Case } 4 .
\end{aligned}
$$

Again, adjusting $\left(K_{2} L\right)_{\text {dipole, mean }}$ to minimize $\chi$, we obtain $\left(K_{2} L\right)_{\text {dipole, mean }} \approx-0.055 \mathrm{~m}^{-2}$ for both cases. The values of $\left(K_{2} L\right)_{\text {dipole, mean }}$ from the Blue and Yellow injection beam-based $Q^{\prime}$ modeling are very close.

\subsection{At the Blue ring store}

At $250 \mathrm{GeV}$ Au store, the $\beta^{*}$ s are $0.85 \mathrm{~m}$ at IP6 and IP8, $5 \mathrm{~m}$ at IP10, IP2, and IP4, and $7 \mathrm{~m}$ at IP12. The natural linear chromaticities are about -83. Tab. 4 shows two cases of $Q_{x, y}^{\prime}$ measurements and modeling, and we have

$$
\begin{aligned}
& Q_{x, y, \text { meas. }}^{\prime} \approx Q_{x, y, \text { model }, \text { no-b2 }}^{\prime} \pm 3.5, \text { for } \text { Case } 5 . \\
& Q_{x, y, \text { meas. }}^{\prime} \approx Q_{x, y, \text { model }, \text { no-b2 }}^{\prime} \pm 3.1, \text { for } \text { Case } 6 .
\end{aligned}
$$

Manipulating $\left(K_{2} L\right)_{\text {dipole, mean }}$ to minimize $\chi$, we derive $\left(K_{2} L\right)_{\text {dipole, mean }} \approx 0.0095 \mathrm{~m}^{-2}$, which is about six times smaller than that from the injection, and has a different sign.

\subsection{At the Yellow ring store}

Tab. 5 shows the $Q^{\prime}$ s measurements and modeling at the Yellow ring store. According to this table, both $Q_{x, y, \text { meas. }}^{\prime}$ are larger than their predictions without sextupole components in the arc main dipoles. This behavior is unlike that observed at the Blue ring injection and store, and Yellow ring at injection, where the $Q_{x, y}^{\prime}$ splits are observed, and furthermore, can be modeled with sextupole components in arc main dipoles.

Since the values for $\left(K_{2} L\right)_{\text {dipole,mean }}$ derived from the Blue and Yellow injections are very close, we choose to adopt $\left(K_{2} L\right)_{\text {dipole,mean }}$ obtained from the Blue store to model $Q^{\prime}$ measurements at the Yellow store. Then, we obtain an empirical equation for the linear chromaticities at the Yellow store,

$$
Q_{x, y, \text { meas. }}^{\prime} \approx Q_{x, y, \text { model }, b 2}^{\prime}+6 .
$$

Here, $\left(K_{2} L\right)_{\text {dipole,mean }}=0.0095 \mathrm{~m}^{-2}$ is used. The source of the 6 unit shift-up in both horizontal and vertical linear chromaticities is unknown. They may be due to various sources. In this Au 2007 run, the IR nonlinear corrections are not applied. 
Table 2: Blue injection: Linear chromaticity measurements and modeling.

\begin{tabular}{|c|c|}
\hline $\begin{array}{l}\text { Case 1: } \\
\text { Tunes }\left(Q_{x}, Q_{y}\right) \\
\text { Chromtic sextupole strengths } \\
\left(Q_{x}^{\prime}, Q_{y}^{\prime}\right) \text { Measured } \\
\left(Q_{x}^{\prime}, Q_{y}^{\prime}\right) \text { from modeling: } \\
\text { with }\left(K_{2} L\right)_{\text {dipole,mean }}=0 \mathrm{~m}^{-2} \\
\text { with }\left(K_{2} L\right)_{\text {dipole,mean }}=-0.054 \mathrm{~m}^{-2}\end{array}$ & $\begin{array}{c}(28.224,29.238) \\
\text { SF: 0.1769m } \mathrm{m}^{-3}, \mathrm{SD}:-0.1606 \mathrm{~m}^{-3} \\
(-8.1,-7.1) \\
(11.7,-27.4) \\
(-8.4,-8.8)\end{array}$ \\
\hline $\begin{array}{l}\text { Case 2: } \\
\text { Tunes }\left(Q_{x}, Q_{y}\right) \\
\text { Chromtic sextupole strengths } \\
\left(Q_{x}^{\prime}, Q_{y}^{\prime}\right) \text { Measured } \\
\left(Q_{x}^{\prime}, Q_{y}^{\prime}\right) \text { from modeling: } \\
\text { with }\left(K_{2} L\right)_{\text {dipole,mean }}=0 \mathrm{~m}^{-2} \\
\text { with }\left(K_{2} L\right)_{\text {dipole,mean }}=-0.047 \mathrm{~m}^{-2}\end{array}$ & $\begin{array}{c}(28.230,29.222) \\
\text { SF: 0.1896m }{ }^{-3}, \mathrm{SD}:-0.1968 \mathrm{~m}^{-3} \\
(-2.9,-3.6) \\
(14.3,-21.4) \\
(-3.2,-5.3)\end{array}$ \\
\hline
\end{tabular}

Table 3: Yellow injection: Linear chromaticity measurements and modeling.

\begin{tabular}{|c|c|}
\hline $\begin{array}{l}\text { Case 3: } \\
\text { Tunes }\left(Q_{x}, Q_{y}\right) \\
\text { Chromtic sextupole strengths } \\
\left(Q_{x}^{\prime}, Q_{y}^{\prime}\right) \text { Measured } \\
\left(Q_{x}^{\prime}, Q_{y}^{\prime}\right) \text { from modeling: } \\
\text { with }\left(K_{2} L\right)_{\text {dipole, } \text { mean }}=0 \mathrm{~m}^{-2} \\
\text { with }\left(K_{2} L\right)_{\text {dipole,mean }}=-0.055 \mathrm{~m}^{-2}\end{array}$ & $\begin{array}{c}(28.231,29.217) \\
\text { SF: } 0.1769 \mathrm{~m}^{-3}, \mathrm{SD}:-0.1606 \mathrm{~m}^{-3} \\
(-6.4,-6.9) \\
(13.5,-27.3) \\
(-6.3,-8.8) \\
\end{array}$ \\
\hline $\begin{array}{l}\text { Case } 4: \\
\text { Tunes }\left(Q_{x}, Q_{y}\right) \\
\text { Chromtic sextupole strengths } \\
\left(Q_{x}^{\prime}, Q_{y}^{\prime}\right) \text { Measured } \\
\left(Q_{x}^{\prime}, Q_{y}^{\prime}\right) \text { from modeling: } \\
\text { with }\left(K_{2} L\right)_{\text {dipole,mean }}=0 \mathrm{~m}^{-2} \\
\text { with }\left(K_{2} L\right)_{\text {dipole,mean }}=-0.055 \mathrm{~m}^{-2}\end{array}$ & $\begin{array}{c}(28.230,29.216) \\
\text { SF: } 0.1876 \mathrm{~m}^{-3}, \mathrm{SD}:-0.1737 \mathrm{~m}^{-3} \\
(-4.1,-5.2) \\
(15.5,-25.26) \\
(-4.0,-7.1)\end{array}$ \\
\hline
\end{tabular}

\subsection{Summary of beam-based modeling}

From these beam-based $Q^{\prime}$ measurements and modeling, there are about 20 unit $Q^{\prime}$ split observed at the $\mathrm{RHIC}$ injection. If we assume they are solely contributed by the sextupole components in the arc main dipoles, we obtain the average strength of these sextupole multipoles in the arc main dipoles $\left(K_{2} L\right)_{\text {dipole,mean }} \approx$ $-0.0505 \mathrm{~m}^{-2}$.

At the Blue ring store, there are about 3.5 unit $Q^{\prime}$ split in the measured $Q_{x, y, \text { meas. }}^{\prime}$, giving $\left(K_{2} L\right)_{\text {dipole,mean }} \approx$ $0.0095 \mathrm{~m}^{-2}$. The $\left(K_{2} L\right)$ dipole, mean derived at the Blue store is about 6 times smaller than that from the injection. Also, the sign of $\left(K_{2} L\right)_{\text {dipole,mean }}$ at the Blue store is opposite to that at the Blue injection.

For the Yellow ring store, we couldn't model the measured chromaticity shifts with sextupole multipoles in the center of arc main dipoles because both measured chromaticities $Q_{x, y}^{\prime}$ are higher than their predictions from the bare optics. If we adopt the value of $\left(K_{2} L\right)_{\text {dipole,mean }}$ obtained from the modeling at the Blue ring store, we get about +6 unit shift-up for both horizontal and vertical chromaticities. We do not know the reason underlying this.

\section{Comparing to bench measurements of magnetic field}

In this section, we compare the sextupole components in the arc main dipoles from the above beam-based modeling with those from the bench measurements of magnetic field. 
Table 4: Blue store: Linear chromaticity measurements and modeling.

\begin{tabular}{|c|c|}
\hline $\begin{array}{l}\text { Case 5: } \\
\text { Tunes }\left(Q_{x}, Q_{y}\right) \\
\text { Chromtic sextupole strengths } \\
\left(Q_{x}^{\prime}, Q_{y}^{\prime}\right) \text { Measured } \\
\left(Q_{x}^{\prime}, Q_{y}^{\prime}\right) \text { from modeling: } \\
\text { with }\left(K_{2} L\right)_{\text {dipole, } \text { mean }}=0 \mathrm{~m}^{-2} \\
\text { with }\left(K_{2} L\right)_{\text {dipole, } \text { mean }}=0.0095 \mathrm{~m}^{-2} \\
\end{array}$ & $\begin{array}{c}(28.228,29.215) \\
\text { SF: } 0.3310 \mathrm{~m}^{-3}, \mathrm{SD}:-0.6575^{-3} \\
(4.0,4.3) \\
(0.8,8.2) \\
(4.0,5.1) \\
\end{array}$ \\
\hline $\begin{array}{l}\text { Case 6: } \\
\text { Tunes }\left(Q_{x}, Q_{y}\right) \\
\text { Chromtic sextupole strengths } \\
\left(Q_{x}^{\prime}, Q_{y}^{\prime}\right) \text { Measured } \\
\left(Q_{x}^{\prime}, Q_{y}^{\prime}\right) \text { from modeling: } \\
\text { with }\left(K_{2} L\right)_{\text {dipole, } \text { mean }}=0 \mathrm{~m}^{-2} \\
\text { with }\left(K_{2} L\right)_{\text {dipole, } \text { mean }}=0.0095 \mathrm{~m}^{-2} \\
\end{array}$ & $\begin{array}{c}(28.232,29.227) \\
\text { SF: } 0.3202 \mathrm{~m}^{-3}, \mathrm{SD}:-0.6348 \mathrm{~m}^{-3} \\
(1.5,1.6) \\
(-1.2,5.1) \\
(1.9,2.0) \\
\end{array}$ \\
\hline
\end{tabular}

Table 5: Yellow store: linear chromaticity measurement and modeling.

\begin{tabular}{l|c}
\hline \hline Case 7: & $(28.232,29.218)$ \\
Tunes $\left(Q_{x}, Q_{y}\right)$ & $(2.3,1.7)$ \\
Chromtic sextupole strengths & SF: $0.2877 \mathrm{~m}^{-3}, \mathrm{SD}-0.5723^{-3}$ \\
$\left(Q_{x}^{\prime}, Q_{y}^{\prime}\right)$ Measured & $(-7.7,-1.2)$ \\
$\left(Q_{x}^{\prime}, Q_{y}^{\prime}\right)$ from modeling: & $(-4.5,-4.2)$ \\
with $\left(K_{2} L\right)_{\text {dipole } \text { mean }}=0 \mathrm{~m}^{-2}$ & \\
with $\left(K_{2} L\right)_{\text {dipole, } \text { mean }}=0.0095 \mathrm{~m}^{-2}$ & $(28.236,29.226)$ \\
\hline Case $8:$ & $(1.1,0.14)$ \\
Tunes $\left(Q_{x}, Q_{y}\right)$ & \\
Chromtic sextupole strengths & SF: $0.2829 \mathrm{~m}^{-3}, \mathrm{SD}-0.5583 \mathrm{~m}^{-3}$ \\
$\left(Q_{x}^{\prime}, Q_{y}^{\prime}\right)$ Measured & $(-8.7,-3.4)$ \\
$\left(Q_{x}^{\prime}, Q_{y}^{\prime}\right)$ from modeling: & $(-5.5,-6.5)$ \\
with $\left(K_{2} L\right)_{\text {dipole, } \text { mean }}=0 \mathrm{~m}^{-2}$ & \\
with $\left(K_{2} L\right)_{\text {dipole,mean }}=0.0095 \mathrm{~m}^{-2}$ & \\
\hline \hline
\end{tabular}

\subsection{Conventions in magnetic field measurement}

In the convention of the RHIC magnet field measurement, the integrated magnet field multipoles are $[3,4]$

$$
\left(B_{y} L\right)+i\left(B_{x} L\right)=\left[B\left(R_{r e f}\right) L\right]\left[10^{-4} \sum_{n=0}^{N_{\max }}\left(b_{n}+i a_{n}\right) \frac{(x+i y)^{n}}{R_{r e f}^{n}}\right] .
$$

Here, $b_{n}$ and $a_{n}, n=0,1,2, \ldots$, are the measured normal and skew multipole field coefficients. $n=0$ represents the dipole field. $B\left(R_{r e f}\right)$ is the main field at radius $R_{r e f}=25 \mathrm{~mm}$ for the arc dipoles. $L$ is the magnet's length. $10^{-4}$ in Eq. (8) is used for normalization, for example, for the arc main dipole magnets, $b_{0}=10^{4}$.

According to Eq. (8), for an arc main dipole magnet, its normal sextupole component is

$$
\left[\left(B_{y} L\right)+i\left(B_{x} L\right)\right]_{\text {sextupole }}=\left[B\left(R_{r e f}\right) L\right] \times 10^{-4} \times b_{2} \times \frac{(x+i y)^{2}}{R_{r e f}^{2}} .
$$


Then, the sextupole's integrated strength is

$$
\begin{aligned}
\left(K_{2} L\right) & =\frac{1}{(B \rho)} \frac{\partial^{2} B_{y}}{\partial x^{2}} \times L \\
& =\frac{\left[B\left(R_{r e f}\right) L\right]}{(B \rho)} \times 10^{-4} \times b_{2} \times \frac{2}{R_{r e f}^{2}} \\
& =\Delta \theta_{\text {bending }} \times 10^{-4} \times b_{2} \times \frac{2}{R_{r e f}^{2}} .
\end{aligned}
$$

Here, $(B \rho)$ is the magnet rigidity, and $\Delta \theta_{\text {bending }}$ is the horizontal bending angle given by one arc main dipole. For the RHIC arc main dipole, $L=9.440656 \mathrm{~m}, \Delta \theta_{\text {bending }}=0.038924 \mathrm{rad}$, then we have

$$
\left(K_{2} L\right)=1.245 \times 10^{-2} \times b_{2} \quad \mathrm{~m}^{-2} .
$$

In Eq. (11), we assumed $b_{2}$ is constant from end to end in the dipole. Eq. (11) is applicable at the injection and the store.

\subsection{Results of bench measurements of magnetic field}

About $20 \%$ of the RHIC arc main dipoles were bench measured in the superconducting state before going into the RHIC tunnel. Integrated field harmonics were measured in these magnets at three currents, 600A, $1450 \mathrm{~A}$ and 5000A. Field harmonics also were measured at a central $1 \mathrm{~m}$-long section at about 35 currents, from $50 \mathrm{~A}$ to $6000 \mathrm{~A}$. Furthermore, $100 \%$ of the magnets were measured warm at a current of $30 \mathrm{~A}$.

In the RHIC $2007 \mathrm{Au}$ run, the main dipole currents at the injection and the store are $473 \mathrm{~A}$ and $5041 \mathrm{~A}$, respectively. First, correlations between warm data and cold data are derived as a function of current, based on measurements at the same 1m-long section of each magnet. The correlation is interpolated for the actual injection and store currents. This warm-cold shift then is applied to the mean values of body harmonics measured warm in $100 \%$ of the arc main dipoles. This gives an estimate of the mean body $b_{2}$ at the current of interest for $100 \%$ of the magnets.

To obtain the integral $b_{2}$ values, the contributions from the magnet ends must be added. Warm-cold correlation for the ends are available only at $660 \mathrm{~A}, 1450 \mathrm{~A}$ and 5000A. However, the current dependence of this correlation for ends is rather weak, and magnet-to-magnet variation is small. Hence, we use the mean values measured at $660 \mathrm{~A}$ and $5000 \mathrm{~A}$ for the end harmonics.

Tab. 7 shows the $b_{2}$ s for the RHIC arc main dipoles predicted from the bench measurements. For the body, $b_{2}$ is assumed constant from end to end. For the lead and return ends, the $b_{2}$ s are normalized by the dipole's length. For example, at the injection, the integrated $b_{2}$ s for lead and return ends are $2.0 \times L$ and $0.42 \times L$, respectively.

Table 6: $b_{2}$ estimates from the bench measurements of magnetic field.

\begin{tabular}{lcccc}
\hline \hline Dipole current $(\mathrm{A})$ & $b_{2}($ body $)$ & $b_{2}($ lead end $)$ & $b_{2}($ return end $)$ & $b_{2}$ ( total $)$ \\
\hline $473($ at injection $)$ & -7.81 & 2.00 & 0.42 & -5.39 \\
5041 (at store $)$ & -2.81 & 2.37 & 0.64 & 0.20 \\
\hline \hline
\end{tabular}

\subsection{Comparing to $\left(K_{2} L\right)_{\text {dipole, mean }}$ from $Q^{\prime}$ modeling}

Based on the linear chromaticity measurements, the linear chromaticity split is about 20 units at injection and 3.5 units at the store. With the offline model, the tune splits can be reproduced with sextupole multipoles in the centers of arc main dipoles. The averaged strengths for these sextupole multipoles are modeled about $-0.0505 \mathrm{~m}^{-2}$ at injection, and about $0.0095 \mathrm{~m}^{-2}$ at the store. From Eq. (8), they are equivalent to -4.06 and 0.76 in $b_{2}$ units at injection and store, respectively.

With this offline optics model, numerically, we have

$$
\left|\Delta Q_{x, y, i n j .}^{\prime}\right| \approx \frac{20}{0.0505} \times\left(K_{2} L\right)_{\text {dipole }, \text { mean }}=396\left(K_{2} L\right)_{\text {dipole }, \text { mean }}
$$

or,

$$
\left|\Delta Q_{x, y, i n j .}^{\prime}\right| \approx \frac{20}{4.06} \times b_{2} \approx 4.9 b_{2}
$$


At the Blue store, we have

$$
\left|\Delta Q_{x, y, \text { store }}^{\prime}\right| \approx \frac{3.5}{0.0095} \times\left(K_{2} L\right)_{\text {dipole }, \text { mean }}=368\left(K_{2} L\right)_{\text {dipole }, \text { mean }},
$$

or,

$$
\left|\Delta Q_{x, y, \text { store }}^{\prime}\right| \approx \frac{3.5}{0.76} \times b_{2} \approx 4.6 b_{2} .
$$

From Tab. 7, the total $b_{2}$ from simply summing up these values from the body, lead and return ends are about -5.39 units at injection, and about 0.20 units at the store. The differences from that from the beam-based modeling are about 1.33 and 0.56 units at the injection and the store, respectively. According to Eqs. (13) and (15), the differences give about 6.5 and $2.6 Q^{\prime}$ splits at injection and store, respectively.

\subsection{Validity of the offline model}

In the above beam-based $Q^{\prime}$ modeling, we assumed the $Q^{\prime}$ splits are solely due to the sextupole components in the arc main dipoles. And with the offline model with sextupole multipoles in the centers of arc main dipoles, the $Q^{\prime}$ splits were reproduced. The linear chromaticities can be predicted very well with $\left(K_{2} L\right)_{\text {dipole,mean }}$ at the Blue ring store, with less than 1 unit error in predication.

In fact, to yield the same amount of shift in horizontal and vertical linear chromaticity from the sextupole multipoles in the centers of the arc main dipoles, the $\beta_{x}$ and $\beta_{y}$ at the center of the arc main dipoles should be equal, or comparable. From the bare optics model, at the center of the arc main dipole,

$$
\frac{\beta_{x}}{\beta_{y}} \approx \frac{23.0}{21.8} \approx 1.06
$$

which is close to 1 .

Strictly, the shifts in linear chromaticities from the sextupole components in the arc main dipoles are

$$
\begin{aligned}
\Delta Q_{x}^{\prime} & =-\frac{N_{\text {dipole }}}{4 \pi}<K_{2} D_{x} \beta_{x}>_{\text {dipole }} L, \\
\Delta Q_{y}^{\prime} & =\frac{N_{\text {dipole }}}{4 \pi}<K_{2} D_{x} \beta_{y}>_{\text {dipole }} L .
\end{aligned}
$$

$N_{\text {dipole }}=144$ is the number of the arc main dipoles. Assuming $K_{2}$ is constant from end to end in the dipole,

$$
\begin{gathered}
\Delta Q_{x}^{\prime}=-\frac{N_{\text {dipole }}}{4 \pi}\left(K_{2} L\right)<D_{x} \beta_{x}>_{\text {dipole, body }}, \\
\Delta Q_{y}^{\prime}=\frac{N_{\text {dipole }}}{4 \pi}\left(K_{2} L\right)<D_{x} \beta_{y}>_{\text {dipole,body }} .
\end{gathered}
$$

At the injection, the ratio of the averaged $\left\langle D_{x} \beta_{x}\right\rangle_{\text {dipole, body }}$ and $\left\langle D_{x} \beta_{y}\right\rangle_{\text {dipole, body }}$ is

$$
\frac{<D_{x} \beta_{x}>_{\text {dipole, body }}}{<D_{y} \beta_{y}>_{\text {dipole, body }}} \approx \frac{33.3}{29.2} \approx 1.14
$$

so giving almost same $Q^{\prime}$ shifts in the horizontal and vertical linear chromaticities.

Substituting $<D_{x} \beta_{x}>_{\text {dipole,body }}$ and $<D_{y} \beta_{y}>_{\text {dipole,body }}$ into Eqs. (19) and (20), we obtain

$$
\left|\Delta Q_{x, y, \text { inj. }}^{\prime}\right| \approx 358\left(K_{2} L\right)_{\text {dipole,mean }},
$$

or, in $b_{2}$,

$$
\left|\Delta Q_{x, y, i n j .}^{\prime}\right| \approx 4.4 b_{2} .
$$

Eqs. (22) and (23) are comparable to Eqs. (12) and (13) that from the numerical modeling, respectively.

In summary, if we assume that the sextupole component is constant in the dipole, the measured $Q^{\prime}$ splits can be equivalently modeled with the sextupole multipoles in the centers of arc main dipoles. 


\subsection{Effects of the dipole ends}

If the contributions to $Q^{\prime}$ s from $b_{2}$ s at dipole ends cannot be ignored, and/or $D_{x} \beta_{x}$ and $D_{x} \beta_{y}$ vary greatly from end to end, the contributions to $Q^{\prime}$ s from the dipole body and the two ends must be evaluated separately. Tab. 8 lists the optics parameters from the bare optics model and the integrated $b_{2}$ s from bench measurements.

It is apparent from Tab. 8 that, at the injection, the contribution to $Q^{\prime}$ s from the return end is smaller than that from the body and the lead end. The contribution to $Q^{\prime}$ s from the lead end is about one third of that from the body. Further, according to Tab. 8 , the signs of the $b_{2} \mathrm{~s}$ for the body and the lead end are opposite.

The RHIC arc main dipoles in each ring were installed with all the lead ends pointing to the same direction. Considering asymmetric $\beta$ functions on both sides of the IPs, averaged along the whole ring, the contributions from lead or return ends to the horizontal and vertical linear chromaticities should be approximately same. Hence, their contributions to $Q^{\prime}$ s from the lead and return ends also should be equivalently modeled with the sextupole multipoles in the centers of arc main dipoles.

Table 7: At injection: Optics parameters and $b_{2}$ s measured at the dipole ends and averaged along the dipole body at injection

\begin{tabular}{lcccc}
\hline \hline Parameters & $D_{x} \beta_{x}$ & $D_{x} \beta_{y}$ & $\left(D_{x} \beta_{x}\right) /\left(D_{x} \beta_{y}\right)$ & Integrated $b_{2}$ \\
\hline one dipole end & 50.3 & 20.6 & $2.4: 1$ & 4.0 (return) \\
the other dipole end & 13.2 & 38.5 & $1: 2.9$ & 18.9 (lead) \\
averaged in the dipole body & 33.3 & 29.2 & 1.1 & -73.7 \\
\hline \hline
\end{tabular}

At the store, the $b_{2} \mathrm{~s}$ in the magnet's body and lead end almost cancel out each other. The total $b_{2}$ is much smaller than that at the injection.

\subsection{Updating the offline model}

Here, we update our offline optics model by appending two extra sextupole multipoles to both ends of each arc main dipole to evaluate the $Q^{\prime}$ contributions from the arc ends. At injection, according Eq. (11) and Tab. 7, from the $b_{2}$ estimates from magnet bench measurements, $\left(K_{2} L\right)_{\text {dipole, bench, body }}=-0.097 \mathrm{~m}^{-1}$, $\left(K_{2} L\right)_{\text {dipole,bench,lead }}=0.025 \mathrm{~m}^{-1}$, and $\left(K_{2} L\right)_{\text {dipole,bench,return }}=0.005 \mathrm{~m}^{-1}$.

Tab. 9 shows the calculated $Q^{\prime}$ shifts with the updated offline model, for Case 1 . The $b_{2}$ s estimated from the bench measurements give about \pm 26.4 units of $Q_{x, y}^{\prime}$ split. From the beam measurement, the $Q_{x, y}^{\prime}$ split is $\sim \pm 20$ units. 6 units in the $Q^{\prime}$ split is equivalent to 1.2 unit change in $b_{2}$, which is in agreement with $\Delta b_{2} \approx 1.3$ from the rough estimate based on the earlier offline model in section 3.3.

Table 8: Blue injection (Case 1): Linear chromaticity measurement and modeling with the updated offline model.

\begin{tabular}{l|cc}
\hline \hline Simulation condition & $\left(Q_{x}^{\prime}, Q_{y}^{\prime}\right)$ from model & $\left(\Delta Q_{x}^{\prime}, \Delta Q_{y}^{\prime}\right)$ \\
\hline without $\left(K_{2} L\right)$ in arc main dipoles & $(11.6,-27.4)$ & - \\
only with $\left(K_{2} L\right)_{\text {dipole, bench, body }}$ & $(-24.1,6.2)$ & $(-35.7,33.6)$ \\
only with $\left(K_{2} L\right)_{\text {dipole, bench, lead }}$ & $(23.3,-36.3)$ & $(11.7,-8.9)$ \\
only with $\left(K_{2} L\right)_{\text {dipole, bench, return }}$ & $(9.3,-25.6)$ & $(-2.3,2.1)$ \\
with all above & $(-14.7,-0.9)$ & $(-26.3,26.5)$ \\
\hline \hline
\end{tabular}

\subsection{Summary of $b_{2}$ comparison}

In this section, we compared the mean sextupole component strength $\left(K_{2} L\right)_{\text {dipole, mean }}$ from the beam-based $Q^{\prime}$ modeling to the $b_{2}$ estimates from the bench measurements. The discrepancies are about 1.3 unit in $b_{2}$ at the injection, and about 0.56 unit in $b_{2}$ at the store, even with the updated offline optics model. They yield about 6 and $2.6 Q^{\prime}$ splits at the injection and the store, respectively.

There are several possible reasons for the discrepancies in the above comparison. For example, at injection, the persistent current effect is not included in the $b_{2}$ estimates. According to Ref. [5], this effect easily 
accounts for a 1.3 unit change in $b_{2}$ at injection. Similarly, for the store, out of the discrepancy of 0.56 units in $b_{2}$, about 0.3 units could come from the uncertainty in the warm-cold correlation. Besides the errors in $b_{2}$ estimates, the deviations in the beam optics and other unknown sextupole components, for example, those in the interaction regions, also could contribute to the discrepancies.

\section{Conclusion}

The measured linear chromaticity splits are modeled with the sextupole components in the arc main dipoles. The equivalent $\left(K_{2} L\right)_{\text {dipole, mean }} \mathrm{s}$ for the sextupole multipoles artificially placed in the center of arc main dipoles are derived with the offline optics model for the Blue ring injection, Blue ring store and Yellow ring injection. They are compared to the $b_{2}$ estimates from the magnet field's bench measurements. The possible reasons for the discrepancies are discussed. With the derived $\left(K_{2} L\right)_{\text {dipole,mean }}$ at the Blue ring store, the linear chromaticities can be predicted with the offline model with error less than 1 unit. At the Yellow ring store, the origin of the +6 unit shift-ups in both horizontal and vertical linear chromaticities is not understood.

\section{Acknowledgments}

We would like to thank S. Peggs, V. Litvinenko and T. Satogata for stimulating discussions during this work. We also thank F. Pilat from the RHIC Accelerator Physics EXperiment (APEX) Committee and $2007 \mathrm{Au}$ run coordinator, and A. Drees for providing us the beam time for this study.

\section{References}

[1] W. Fischer, A. Jain and S. Tepikian, PRST-AB, 4, 041002 (2001).

[2] N. Maltisky, private communications, 2007.

[3] A. Jain, D. Trbojevic, F. Dell, S. Peggs, P. Wanderer and J. Wei, BNL RHIC/AP/95, August, 1996.

[4] S. Trahern and F. Pilat, BNL RHIC/AP/99, June, 1996.

[5] A. Jain, G. Ganetis, J. Muratore, R. Thomas and P. Wanderer, BNL Magnet Division Note 593-11 (AM-MD-294), May 10, 2000.

http://www.bnl.gov/magnets/magnet_files/Publications/FastMeasurements.pdf . 